\title{
SOME RECENT INVESTIGATIONS CONCERNING THE SECTIONS OF POWER SERIES AND RELATED DEVELOPMENTS*
}

\author{
BY GABRIEL SZEGÖ
}

1. Introduction. The definition of analytic functions given by Weierstrass is based primarily on the notion of power series. Numerous results and questions of the modern theory of these functions can be formulated as definite relations between the coefficients of a power series and the values of the corresponding function inside or outside of the circle of convergence or on this circle itself. Analogous questions may be raised concerning trigonometric series. In what follows a short report of some recent investigations in this direction will be given. The vastness of this field makes it impossible to be complete. Thus we point out mainly the typical methods used and the most characteristic results. $\dagger$

2. Problem. We consider the set or the class of power series

$$
c_{0}+c_{1} z+c_{2} z^{2}+\cdots+c_{n} z^{n}+\cdots
$$

convergent in a circle of the complex $z$ plane (say for $|z|<1$ ) and satisfying some conditions there, say $C$. In a very general formulation the problem is to find necessary and sufficient conditions in terms of the coefficients $c_{n}$ in order that the function $f(z)$ represented by (1) should satisfy the condition $C$. This problem has been solved in a more or less applicable form only for some special classes of power series. In 1911 Carathéodory [1] and Toeplitz [39] obtained a condition of this kind for power series $f(z)$ with a positive real part in the unit circle. Their condition has a "quasi-algebraic" character, containing an infinite number of algebraic conditions. A second very important class, that of power series remaining bounded in the unit circle, $|f(z)| \leqq M$, can be reduced, at least in principle, to the class mentioned

* An address delivered at the meeting of the Society on January 1, 1936, in St. Louis, by invitation of the Program Committee.

$\dagger$ Some of the older results referred to in this paper are contained in a previous report on this subject. See [34] in the list of references given at the end of the paper. 
[Carathéodory and Fejér, 2]. This reduction gives a possibility of characterizing the boundedness property again by some conditions in terms of the coefficients. In 1917 Schur [27] gave a very elegant algebraic form for these conditions.

Apart from these special classes and some other classes more or less connected with them, it is rather hopeless to expect an exhaustive solution of this problem. For instance, we do not know the necessary and sufficient conditions in terms of the coefficients in order that $f(z)$ should be a simple or univalent function in the unit circle. Therefore we confine ourselves to a more special question asking for some properties of the coefficients $c_{n}$ or of the terms $c_{n} z^{n}$ of the power series (1), or more generally of some linear combinations of these terms which are implied by the condition $C$ in question. This problem is not at all trivial even for the classes mentioned before. In principle the algebraic characterization of the coefficients yields, of course, a method of attacking the problem; this, however, only in principle, and the effective determination of some maxima or minima connected with the coefficients can be an extremely difficult algebraic task.

It should be emphasized that historically these problems have originated in some questions concerning ordinary Fourier series. It suffices to mention only two questions of this sort which have become rather classical nowadays. The first is the problem of Lebesgue, to determine the maximum of the $n$th section of the Fourier series of all functions remaining $\leqq 1$ in absolute value.* These maxima, called Lebesgue constants, gained a particular importance through the investigations of Lebesgue, showing that the unboundedness of these constants is the deeper cause of the existence of continuous functions with divergent Fourier series. Another question is Carathéodory's problem of characterizing the Fourier constants of a positive real function, a problem closely related to that mentioned above regarding analytic functions with a positive real part in the unit circle.

\section{Bounded Functions. Coefficients, Partial Sums, and Cesàro}

${ }^{*} \mathrm{H}$. Lebesgue, Sur la divergence et la convergence non-uniforme des séries de Fourier, Comptes Rendus, vol. 141 (1905), pp. 875-877; Leçons sur les Séries Trigonométriques, (1906), art. 45, 46, pp. 86-88. See also A. Haar, Zur Theorie der orthogonalen Funktionensysteme, Mathematische Annalen, vol. 69 (1910), pp. 331-371. 
Means. Returning to the complex problem, let us consider in a more detailed way the set of power series $f(z)$ satisfying the special condition $E:|f(z)| \leqq 1$ in $|z|<1$. For the sake of convenience we choose for the upper bound $M=1$. Cauchy's integral representation of the coefficients furnishes, then, the inequalities $\left|c_{n}\right| \leqq 1$ for all values of $n$. The bound 1 is of course the best possible since $f(z)=1$ satisfies condition $E$. Much more holds, however, namely, that the sum

$$
\left|c_{0}\right|^{2}+\left|c_{1}\right|^{2}+\left|c_{2}\right|^{2}+\cdots+\left|c_{n}\right|^{2}+\cdots
$$

is convergent and also $\leqq 1$. This incidentally is the case for a more extensive class of power series, namely, for the functions $f(z)$ regular in $|z|<1$ and satisfying there the condition

$$
\frac{1}{2 \pi} \int_{-\pi}^{+\pi}\left|f\left(r e^{i \phi}\right)\right|^{2} d \phi \leqq 1, \quad(r<1) .
$$

We return to this class and to related classes in $\$ 11$.

So much about the limitation of the coefficients or, what is the same, of the single terms. The question arises now what can be said about the partial sums or sections

$$
s_{n}(z)=c_{0}+c_{1} z+c_{2} z^{2}+\cdots+c_{n} z^{n}
$$

of a power series of class $E$ if $|z| \leqq 1$.

The exact maximum of $\left|s_{n}(z)\right|$ in $|z| \leqq 1$ uniformly for the whole class $E$ was determined by Landau [16] in the explicit form

$$
1+\sum_{\nu=1}^{n}\left(\frac{1 \cdot 3 \cdots(2 \nu-1)}{2 \cdot 4 \cdots 2 \nu}\right)^{2}
$$

This expression becomes $\infty$ for $n \rightarrow \infty$, a fact which is in close relation to a previous result of Fejer [5], stating the existence of functions with the property $E$ whose partial sums are unbounded in $|z|<1$. In particular, if $f(z)$ has the property $E$ in $|z|<1$, the partial sums do not have the same property necessarily in $|z|<1$.

These results suggest the question of determining the exact maximum or, at any rate, proper upper bounds for a given linear combination

$$
L_{n}(z)=\lambda_{0} c_{0}+\lambda_{1}\left(c_{1} z\right)+\lambda_{2}\left(c_{2} z^{2}\right)+\cdots+\lambda_{n}\left(c_{n} z^{n}\right)
$$


of the first $n+1$ terms of a function $f(z)$ of class $E$; here $z$ ranges over the unit circle, $|z| \leqq 1$. A very important linear combination of this kind has been pointed out by Fejér [6], who proved that the Cesàro means of (1) satisfy the same condition $E$ as the function itself. Denoting these means by $\sigma_{n}(z)$, we have

$$
\begin{aligned}
\sigma_{n}(z) & =\frac{s_{0}(z)+s_{1}(z)+s_{2}(z)+\cdots+s_{n}(z)}{n+1}=\frac{S_{n}(z)}{n+1} \\
& =\sum_{\nu=0}^{n}\left(1-\frac{\nu}{n+1}\right) c_{\nu} z^{\nu},
\end{aligned}
$$

and the inequalities $\left|\sigma_{n}(z)\right| \leqq 1$ hold for $|z| \leqq 1,(n=0,1,2, \cdots)$. This result has, also, a parallel in the theory of Fourier series. It reminds us of the analogous, nowadays classical, property of the Cesàro means of Fourier series likewise due to Fejér.* The converse of the theorem is also true: The function $f(z)$ has the property $E$ in $|z|<1$, if and only if the same is valid for all the Cesàro means $\sigma_{n}(z)$ in $|z| \leqq 1$.

4. Continuation. Linear Combinations. Various methods have been developed in order to obtain proper upper bounds for the linear combination $L_{n}(z)$ in (6) if $f(z)$ is in $E$ and $|z| \leqq 1$.

(a) Landau's method was extended by Szász [31], giving an upper bound for $L_{n}(z)$ in each case, and even obtaining the exact maximum if a certain algebraic condition is satisfied. Assuming $\lambda_{n} \neq 0$ we develop the expression

$$
\left(\lambda_{n}+\lambda_{n-1} x+\lambda_{n-2} x^{2}+\cdots+\lambda_{0} x^{n}\right)^{1 / 2}
$$

(with an arbitrary determination of the square root) in a power series $\mu_{0}+\mu_{1} x+\mu_{2} x^{2}+\cdots$; the finite sum

$$
\left|\mu_{0}\right|^{2}+\left|\mu_{1}\right|^{2}+\left|\mu_{2}\right|^{2}+\cdots+\left|\mu_{n}\right|^{2}
$$

then furnishes the upper bound mentioned. In the particular case when the polynomial $\mu_{0}+\mu_{1} z+\cdots+\mu_{n} z^{n}$ is different from 0 in $|z|<1$, this is the exact maximum in question.

From this general theorem Landau's result $\left(\lambda_{\nu}=1\right)$ and Fejér's result $\left(\lambda_{\nu}=1-\nu /(n+1)\right)$ can be easily obtained.

(b) In a joint investigation with Schur [29], we gave an-

* Untersuchungen über Fouriersche Reihen, Mathematische Annalen, vol. 58 (1904), pp. 51-69; especially p. 60. 
other method, in principle very elementary, of obtaining linear combinations (6) remaining $\leqq 1$ in $|z| \leqq 1$, that is, having the same property $E$ as the function itself. This is, namely, the case if the coefficients $\lambda_{\nu}$ are real, $\lambda_{0}=1$, and the trigonometric polynomial

$$
T(\phi)=\frac{1}{2}+\lambda_{1} \cos \phi+\lambda_{2} \cos 2 \phi+\cdots+\lambda_{n} \cos n \phi
$$

assumes only non-negative values for all real values of $\phi$. This follows readily from the representation

$$
\text { (9) } \quad L_{n}(z)=L_{n}\left(r e^{i \theta}\right)=\frac{1}{\pi} \int_{-\pi}^{+\pi} f\left(r e^{i \phi}\right) T(\theta-\phi) d \phi, \quad(r<1) \text {. }
$$

(c) In several investigations of a related kind a third method has been used, based on Fejér's result concerning the Cesàro means. Introducing in (6) for $c_{\nu} z^{\nu}$ the expression $s_{\nu}(z)-s_{\nu-1}(z)$ and for $s_{\nu}(z)$ the difference $S_{\nu}(z)-S_{\nu-1}(z)$ [see (7)], we obtain, for $n \geqq 2$,

Hence

$$
\begin{aligned}
L_{n}(z)=\sum_{\nu=0}^{n-2}\left(\lambda_{\nu}-\right. & \left.2 \lambda_{\nu+1}+\lambda_{\nu+2}\right) S_{\nu}(z) \\
& +\left(\lambda_{n-1}-2 \lambda_{n}\right) S_{n-1}(z)+\lambda_{n} S_{n}(z)
\end{aligned}
$$

$$
\begin{aligned}
\left|L_{n}(z)\right| \leqq & \sum_{\nu=0}^{n-2}\left|\lambda_{\nu}-2 \lambda_{\nu+1}+\lambda_{\nu+2}\right|(\nu+1) \\
& +\left|\lambda_{n-1}-2 \lambda_{n}\right| n+\left|\lambda_{n}\right|(n+1)
\end{aligned}
$$

follows. This bound in general is not the exact maximum. It is attained by the function $f(z)=\epsilon=$ const., $|\epsilon|=1$, provided the $\lambda_{\nu}$ are all real and the following inequalities hold

$$
\begin{aligned}
\lambda_{\nu}-2 \lambda_{\nu+1}+\lambda_{\nu+2} & \geqq 0, \quad(\nu=0,1,2, \cdots, n-2), \\
\lambda_{n-1}-2 \lambda_{n} & \geqq 0, \quad \lambda_{n} \geqq 0 .
\end{aligned}
$$

The maximum appearing on the right side is then $=\lambda_{0}{ }^{*}$

The same method can be used to prove the non-negativeness of the trigonometric polynomial (8) if the conditions (12) are satisfied [Fejér, 11]. Thus in this case the condition mentioned in (b) is also satisfied.

\footnotetext{
* Concerning this method see in addition Fejér [10].
} 
By means of one or the other of these methods numerous interesting results have recently been obtained for power series of class $E$. A short enumeration of the most typical theorems of this kind will be given now.

5. A Theorem of Fejér and Rogosinski. These authors have proved independently of each other $[7,24]$ that all partial sums of a power series of class $E$ in $|z|<1$ are of class $E$ in $|z|<1 / 2$, that is, they remain $\leqq 1$ in absolute value there. This radius $1 / 2$ is here the best possible; in general it cannot be replaced by a larger one.

This theorem is surprising because in the unit circle itself the partial sums of proper functions of class $E$ become arbitrarily large (see $\S 3$ ).

The proof can be based, for instance, on the method (b) [Landau, 17]. Indeed, the statement is equivalent to

$$
\left|c_{0}+2^{-1} c_{1} z+2^{-2} c_{2} z^{2}+\cdots+2^{-n} c_{n} z^{n}\right| \leqq 1, \quad(|z| \leqq 1) .
$$

Thus we have here $\lambda_{\nu}=2^{-\nu}$ and conditions (12) are satisfied because

$$
\begin{aligned}
\lambda_{\nu}-2 \lambda_{\nu+1}+\lambda_{\nu+2} & =2^{-\nu}-2^{-\nu}+2^{-\nu-2}=2^{-\nu-2}>0, \\
\lambda_{n-1}-2 \lambda_{n} & =2^{-n+1}-2^{-n+1}=0, \quad \lambda_{n}=2^{-n}>0 .
\end{aligned}
$$

Moreover, functions of the type

$$
\frac{z+\alpha}{1+\bar{\alpha} z}=\alpha+\left(1-|\alpha|^{2}\right) z+\cdots
$$

satisfy condition $E$ in $|z|<1$ if $|\alpha|<1$. Choosing any point $t$ with $1 / 2<|t|<1$ and taking $\arg \alpha=\arg t$, we have

$$
\left|\alpha+\left(1-|\alpha|^{2}\right) t\right|=|\alpha|+\left(1-|\alpha|^{2}\right)|t|>1,
$$

or $(1+|\alpha|)|t|>1$ provided that $|\alpha|$ is sufficiently near to 1 .

6. A Theorem of I. Schur and Szegö. In a joint investigation with Schur [29] we noticed the fact that the preceding statement that $1 / 2$ is the "best" constant of the last theorem, is true only if the totality of all partial sums is considered. Leaving out of consideration the section $n=1$ and taking only the sections $s_{n}(z)$ with $n \geqq 2$, a larger circle with the same property, namely, $|z| \leqq(3 / 8)^{1 / 2}=0.6123 \ldots$, can be determined. Here the 
number $(3 / 8)^{1 / 2}$ is again the best possible. This leads to the general problem of determining the greatest circle $|z| \leqq r_{n}$ in which all the partial sums beginning with the $n$th satisfy the same boundedness condition as the function. Jointly with Schur, we gave an algebraic method to calculate those numbers, and proved that $r_{n} \uparrow 1$. A direct computation shows

$$
r_{1}=0.5, r_{2}=0.6123 \cdots, r_{3}=0.6478 \cdots, r_{4}=0.7204 \cdots \text {. }
$$

Furthermore, we found a simple asymptotic formula for $r_{n}$ if $n \rightarrow \infty$,

$$
r_{n}=1-\frac{\log n-\log \log n+\log 2+\epsilon_{n}}{n}, \quad\left(\epsilon_{n} \rightarrow 0\right) .
$$

This means that the "singular" behavior of the $n$th partial sum (to be $>1$ in absolute value) can appear only in a small strip around the unit circle whose width asymptotically $=n^{-1} \log n$ if $n$ is large.

If the partial sums are replaced by the Cesàro means, the corresponding numbers all are $=1$ (see \$3). As a generalization of the partial sums and the Cesàro means the general Cesàro means $s_{n}{ }^{(k)}(z)$ can be now introduced. Thus we can determine a definite circle $|z| \leqq r_{n}^{(k)}$ (the greatest of this kind) in which all the Cesàro means $s_{m}{ }^{(k)}(z),(m \geqq n)$, of all functions $f(z)$ of class $E$ are also of class $E$. Here the order $k$ is between 0 and 1 . The determination or characterization of these numbers $r_{n}{ }^{(k)}$ seems to be difficult. We proved only $[29$, pp. 556-558] that

$$
r_{1}^{(k)}=\frac{k+1}{2} \text {. }
$$

7. A Theorem of Rogosinski and Szegö. Recalling again the fundamental property of the Cesàro means mentioned in $\S 3$, we notice that they are built up from all partial sums from the 0th to the $n$ th. Thus the question arises to find some simple linear combinations of the $n$ first terms expressible by some values of the $n$th partial sum alone, and having the same boundedness property as the Cesàro means or at any rate remaining bounded for $n \rightarrow \infty$. In a joint investigation with Rogosinski [25] we considered expressions of the form

$$
A s_{n}\left(z e^{a / n}\right)+B s_{n}\left(z e^{b / n}\right),
$$


$A, B, a, b$ being given complex constants. The problem is to determine the values of these constants for which this expression remains bounded, uniformly for all $n$, for all $|z| \leqq 1$ and for all functions of class $E$. A necessary and sufficient condition which we obtained for this property is the equation

$$
A e^{a}+B e^{b}=0 \text {. }
$$

The exact bound depends in general on the given constants. The proof of the boundedness can be readily given by means of method (c). The determination of the exact bound in the case (1) below has been carried out by means of (a).

This question has its origin likewise in some analogous problems concerning Fourier series.*

Two special cases should be mentioned:

(1) $a$ and $b$ are both pure imaginary,

(2) $a$ and $b$ are both real.

In case (1) our expression becomes, after multiplication by a proper constant and a proper rotation of the unit circle,

$$
e^{i \alpha} s_{n}\left(z e^{-i \alpha / n}\right)-e^{-i \alpha} s_{n}\left(z e^{i \alpha / n}\right),
$$

$\alpha$ being a fixed real number. Here we obtained for $0<\alpha \leqq \pi / 2$, the exact bound $2 \int_{0}^{\alpha}\left[J_{0}(x)\right]^{2} d x, J_{0}(x)$ being Bessel's function of order $0 . \dagger$ This statement is equivalent to the interesting inequality

$$
\begin{aligned}
\left|\sum_{\nu=0}^{n} \frac{\sin \left(1-\frac{\nu}{n+1}\right) \alpha}{\sin \alpha} c_{\nu} z^{\nu}\right| & <|\sin \alpha|^{-1} \int_{0}^{\alpha}\left[J_{0}(x)\right]^{2} d x \\
& (|z| \leqq 1 ; n=0,1,2, \cdots) .
\end{aligned}
$$

Here the right side cannot be replaced by a smaller number. For $\alpha \rightarrow 0$ we obtain again Fejér's theorem concerning Cesàro means. $\ddagger$

* See W. Rogosinski, Reihensummierung durch Abschnittskoppelungen, Mathematische Zeitschrift, vol. 25 (1926), pp. 132-149. $\alpha>0$.

† In a subsequent paper [36] I proved that this is true for all values of $\alpha$,

$\ddagger$ Szász had previously considered [31] this linear combination and applied method (a) without discussing the question of the maximum. 
We have, for instance, for $\alpha=\pi / 2$,

$$
\begin{aligned}
\frac{1}{2} \mid s_{n}\left(z e^{-i \pi /(2 n)}\right)+ & s_{n}\left(z e^{i \pi /(2 n)}\right) \mid \\
& <\int_{0}^{\pi / 2}\left[J_{0}(x)\right]^{2} d x=1.0777 \cdots .
\end{aligned}
$$

The last number cannot be replaced by a smaller one.

In case (2) we obtain the inequality

$$
\left|s_{n}(\rho z)-\rho^{n} s_{n}(z)\right| \leqq 1-\rho^{n}, \quad(0<\rho<1),
$$

which can be also written in the form

$$
\left|\sum_{\nu=0}^{n} \frac{\rho^{\nu}-\rho^{n}}{1-\rho^{n}} c_{\nu} z^{\nu}\right| \leqq 1, \quad(|z| \leqq 1 ; n=0,1,2, \cdots) .
$$

Hence Fejér's theorem arises again as a limiting case for $\rho \rightarrow 1$. Among others we made the following curious conclusion from this inequality. Supposing $\left|s_{n}(z)\right| \leqq 1$ is true at a point $z=z_{0}$ somewhere in the unit circle for the $n$th section of a fixed function of our class, then the same will be true on the whole segment $O z_{0}$, connecting $z_{0}$ with the origin. Or, the set of all points in the unit circle in which a particular section of a particular function of our class is $\leqq 1$ in absolute value defines a so-called star-shaped region. (Of course this always contains the origin.)

8. Another Average of Sections. A further remarkable property of the partial sums of the same class is that the mean value along any segment starting from the origin

$$
z^{-1} \int_{0}^{z} s_{n}(t) d t, \quad(|z| \leqq 1)
$$

is $\leqq 1$ in absolute value, or it belongs to the same class as the function $f(z)$ itself [25]. This property is not at all trivial; it depends on the non-negativeness of the trigonometric polynomials

$$
\frac{1}{2}+\frac{1}{2} \cos \phi+\frac{1}{3} \cos 2 \phi+\cdots+\frac{1}{n+1} \cos n \phi,
$$

the proof of which is rather troublesome $[25, \S 5]$. (The method 
of Fejér [11] needs here an essential modification.) From this fact the statement follows easily by means of method (b).

9. Harmonic Functions. If the condition $|f(z)| \leqq 1$ is replaced by $R f(z)>0$, analogous properties can be studied. If the function is normalized by the condition $R f(0)=1$, the exact inequalities $\left|c_{n}\right| \leqq 2,(n=1,2,3, \cdots)$, hold [Carathéodory, 1]. The analog of Landau's problem has been treated by Schur [28]. He obtained for the real part of the $n$th section a minimal value in $|z| \leqq 1$ which divided by $n$ tends to $\min (2 \sin x / x)$ for $n \rightarrow \infty$ (provided that the normalization $R f(0)=1$ has been made). Moreover the theorem in $\$ 5$ concerning the circle $|z| \leqq 1 / 2$ remains valid and the assertion about the numbers $r_{n}$ of $\$ 6$ can similarly be taken over word for word. The inequalities (21) and (23) have likewise their exact analogs for this class.

The theory of analytic functions with a positive real part is equivalent to that of positive harmonic functions. Thus the preceding results can be formulated also in terms of positive harmonic functions regular in the unit circle. If this is done the formulation of corresponding questions in space for developments in terms of spherical harmonics becomes possible. The analog of Schur's theorem formulated above holds for these developments [Szegö, 33, §4] with $n$ replaced by $n^{2}$ and the minimal value by $\min \left(2 J_{1}(x) / x\right)$, where $J_{1}(x)$ denotes Bessel's function of order 1 . Instead of $r_{1}=1 / 2$ we obtain in this threedimensional case the number $r_{1}=1 / 3$ [Szegö, 33, §5], a result connected with an older remark of Pick [22],* and we can introduce the analog of the numbers $r_{n}$ too. The exact determination of these new numbers $r_{n}$ and the proof that they increase seems, however, to be extremely complicated. The asymptotic determination is still possible in a form somewhat different from that in the two-dimensional case. I have found recently [37]

$$
r_{n}=1-\frac{2 \log n-\log \log n+\gamma+\epsilon_{n}}{n}, \quad\left(\epsilon_{n} \rightarrow 0\right) .
$$

* Montel has recently published [Comptes Rendus, vol. 201 (1935), pp. 119-121] a note on this subject, rediscovering Pick's theorem as well as related estimates for the $n$th term of the development in spherical harmonics [Szegö, 33, §3]. He does not refer to the older literature. 
Here $\gamma=\log 2$ if $n$ is odd, and $\gamma=\log (2 \mu)$ if $n$ is even, while $\mu=0.4028 \cdots$ denotes the maximum of $-J_{0}(x)$ for all real values of $x$.

10. Polynomials. Various extremal problems treated before can be sharpened if we consider instead of the whole class $|f(z)| \leqq 1$ or $R f(z)>0$ the subset of polynomials of a given degree $N$ satisfying one of these conditions in the unit circle. The same is true for developments in terms of spherical harmonics. In case of functions with a positive real part Carathéodory's inequalities (see $\$ 9$ ) can be replaced by

$$
\begin{aligned}
\left|c_{n}\right| \leqq 2 \cos \left(\frac{\pi}{\left[\frac{N}{n}\right]+2}\right) & , \\
& \left(n=1,2, \cdots, N ; c_{0}=1\right)
\end{aligned}
$$

[Egerváry, Lǔkács, and Szász; see Szegö, 33, pp. 624-626]. Sidon [30] observed for the same class that the circle $|z| \leqq[2 \cos (\pi /(N+2))]^{-1}$, instead of $|z| \leqq 1 / 2$, has the property mentioned in \$9. Recently Levin [18] has obtained several results in this direction for polynomials belonging to class $E$. As regards three-dimensional problems of this kind see Szegö [33, pp. 626-632].

11. Mean Value Conditions. A rather obvious generalization of the boundedness property is the restriction that the integral mean value

$$
\left\{\frac{1}{2 \pi} \int_{-\pi}^{+\pi}\left|f\left(r e^{i \phi}\right)\right| p d \phi\right\}^{1 / p}
$$

remain bounded for $r<1, f(z)$ being a given function regular for $|z|<1$. Here $p$ denotes a fixed positive number. This condition, assuming the bound to be equal to 1 , defines a class $E_{p}$. The limiting case $E_{\infty}$ is the class $E$ discussed before. The class $E_{2}$ can be characterized by the condition that the sum (2) of squares of the coefficients is convergent and $\leqq 1$. Therefore in this case it is not difficult to carry out an algebraic treatment of various questions concerning the terms, partial sums, linear combinations of terms, and so on. 
More difficult is the treatment of the class $E_{1}$ corresponding to Hardy's mean value.* Numerous questions concerning coefficients, sections, and related topics can be discussed here by using the following theorem due to Fekete and M. Riesz. $\dagger$

$A$ function $f(z)$ belongs to $E_{1}$ if and only if it can be written in the form $f(z)=g(z) h(z)$, where both functions $g(z)$ and $h(z)$ belong to $E_{2}$.

This leads to the following parametric representation of the coefficients of $f(z)$ :

$$
\begin{aligned}
& c_{n}=u_{0} v_{n}+u_{1} v_{n-1}+\cdots+u_{n} v_{0}, \quad(n=0,1,2, \cdots) . \\
& \sum_{n=0}^{\infty}\left|u_{n}\right|^{2} \leqq 1, \quad \sum_{n=0}^{\infty}\left|v_{n}\right|^{2} \leqq 1 .
\end{aligned}
$$

It is easy to show the existence of some numbers $r_{n}$ depending only on $n$ such that the partial sums of degree $\geqq n$ have a Hardy mean value $\leqq 1$ for $r \leqq r_{n}$ provided that the corresponding mean value of the function in the unit circle is $\leqq 1$, or else such that $s_{m}(z),(m \geqq n)$, belong to class $E_{1}$ in $|z| \leqq r_{n}$ if $f(z)$ belongs to $E_{1}$ in $|z|<1$. The number $r_{n}$ should be here, of course, the largest possible. The determination of these numbers, unlike that in the limiting case $E_{\infty}$, seems here to be rather difficult; their asymptotic determination may be less intricate although it has not been carried through as yet.

Using the representation (28) I have calculated the largest number $r_{0}$ for which

$$
\frac{1}{2 \pi} \int_{-\pi}^{+\pi}\left|s_{1}\left(r_{0} e^{i \phi}\right)\right| d \phi \leqq 1
$$

The result is $r_{0}=\left(2 \mu^{1 / 2}\right)^{-1}=0.9146 \cdots$, where $\mu$ is the maximum of the function

* G. H. Hardy, The mean value of the modulus of an analytic function, Proceedings of the London Mathematical Society (2), vol. 14 (1915), pp. 269-277.

† Verbal communication. The proof can be based on the following facts: (i) Any function $f(z)$ of class $E_{1}$ regular in $|z| \leqq 1$ can be written in the form $f(z)=l(z) q(z), l(z)$ being a rational function regular and with $|l(z)|<1$ in $|z|<1, q(z)$ a regular function of class $E_{1}$ and $\neq 0$ in $|z|<1$. (ii) From any set of functions $\left\{f_{\nu}(z)\right\}$ belonging to $E_{2}$ a subset can be selected which is uniformly convergent in any circle $|z| \leqq r,(r<1)$. Another direct (but less elementary) way would be to make use of the factorization theorem of F. Riesz, Mathematische Zeitschrift, vol. 18 (1923), pp. 87-95. 


$$
x^{-2}\left(\pi^{-1} \int_{0}^{\pi}\left(1+x^{2}-2 x \cos \phi\right)^{1 / 2} d \phi-1\right)
$$

for $x>1$, or the maximum of

$$
(\sin \alpha / 2)^{2}(\cos \alpha / 2)^{-4}\left(E(\sin \alpha)-(\sin \alpha / 2)^{2}\right)
$$

for $0<\alpha<\pi / 2$, and $E(k)$ denotes Legendre's elliptic integral of the second kind

$$
E(k)=\frac{2}{\pi} \int_{0}^{\pi / 2}\left(1-k^{2} \sin ^{2} \phi\right)^{1 / 2} d \phi .
$$

These results show that all these questions have a more complicated character than in the case $E_{\infty}$.

12. Univalent Bounded Functions. A very interesting subset of $E=E_{\infty}$ can be obtained by imposing upon $f(z)$ the additional condition to be univalent or simple in the unit circle $|z|<1$. Fejér [8, see also 9] proved that the partial sums of a power series of this kind remain uniformly bounded in $|z|<1$. He obtained by application of his Cesàro mean theorem the bound $1.7071 \ldots$ Using $\left(20^{\prime}\right)$ I found $[35, \S 3]$ a better estimate $1.6160 \cdots$. Recently Levin $[18, \$ 4]$ succeeded in improving this result; the exact bound, however, is not known as yet.

In all these investigations the univalency of the function is used only to a very moderate extent, namely, in the following form: The area of the $w$-set in the conformal representation $w=f(z)$ corresponding to $|z|<1$ is $\leqq \pi$, whence

$$
\left|c_{1}\right|^{2}+2\left|c_{2}\right|^{2}+3\left|c_{3}\right|^{2}+\cdots+n\left|c_{n}\right|^{2}+\cdots \leqq 1 .
$$

The results mentioned remain valid for the larger subset of $E$ satisfying this condition. Levin investigated [18, §3] some generalizations of this class requiring, besides $E$, the inequality

$$
\alpha_{1}\left|c_{1}\right|^{2}+\alpha_{2}\left|c_{2}\right|^{2}+\cdots+\alpha_{n}\left|c_{n}\right|^{2}+\cdots \leqq 1,
$$

where $\left\{\alpha_{n}\right\}$ is a given sequence of positive numbers.

13. Univalent Functions. Another rather complicated class is that of the univalent functions (without the condition $E$ ). The best known general limitation for the coefficients has been obtained by Littlewood [19, pp. 498-499]. It is: $\left|c_{n}\right|<e n$ provided that $c_{1}=1$. The analog of the numbers $r_{n}$ can be defined 
here as well, requiring univalency of $s_{m}(z),(m \geqq n)$, for $|z|<r_{n}$, but nothing is known about their determination and properties in general, doubtless a rather intricate question. I proved [35] that $r_{1}=1 / 4$, or each section remains univalent in the circle $|z|<1 / 4$, a number which cannot be replaced by a larger one. An asymptotic characterization of the $r_{n}$ for $n \rightarrow \infty$ seems also to present some difficulties; however, these might not be insurmountable.

The methods used in the treatment of these problems are not quite simple. In general, Koebe's distortion theorem* is needed, sometimes even Löwner's theory of univalent functions. $\dagger$

14. Related Classes. Even more difficult is the treatment of "odd" univalent functions, that is, univalent functions of the form (1) with $c_{2}=c_{4}=c_{6}=\cdots=0$. Littlewood and Paley proved [20] that in this case $\left|c_{n}\right|<K, c_{1}=1, K$ being an absolute constant.

The treatment of star-shaped and convex representations is possible by using more elementary methods, primarily Carathéodory's theory of functions with a positive real part.

Various elegant results have been recently found for univalent power series of the form (1) with real coefficients. For instance, the exact inequality $\left|c_{n}\right| \leqq n,(n=2,3,4, \cdots)$, holds in this case, $c_{1}=1$ [Dieudonné, 4; Rogosinski, 26]. More generally, Rogosinski [26] studied power series of the form (1) assuming real values if and only if $z$ itself is real. He obtains a complete characterization of this class (called "typisch-reell") in terms of the coefficients.

Fejér examined [12, pp. 61-62] univalent functions with real coefficients having the property that the image of the unit circle has at most two points of intersections with every straight line parallel to the imaginary axis. The partial sums are in this case univalent in $|z|<1 / 4 \ddagger$ and even in $|z|<3^{-1 / 2}$ if all the $c_{2 \nu}$ disappear.

By generalizing and combining these classes, numerous vari-

* See, for instance, G. Pólya and G. Szegö, A ufgaben und Lehrsätze aus der Analysis, (1925), vol. 2, p. 27, problem 151.

$\dagger \mathrm{K}$. Löwner, Untersuchungen über schlichte konforme Abbildungen des Einheitskreises, Mathematische Annalen, vol. 89 (1923), pp. 103-121.

$\ddagger$ Which is generally true for univalent functions (see above), but it can be proved much more easily in this case. 
ations of the problem regarding the partial sums are possible [see, for instance, Takahashi, 38 and Noshiro, 21].

15. Power Series with Monotonic Coefficients. Finally another point of view likewise appearing in various recent investigations may be mentioned in a few words. Introduction of proper regularity properties for the whole sequence of coefficients is possible. We can expect then an analogous regular behaviour of the partial sums, of the remainder sums, of some linear combination of the terms, and so on. From this point of view Fejér has recently investigated $[13,15]$ trigonometric and power series, the coefficients of which are monotonic of a definite order $k$, that is, having positive 0 th, 1 st, $2 \mathrm{nd}, \cdots, k$ th differences. In this case certain positiveness, monotone character, or convexity properties of the partial sums can be proved. Furthermore, in a joint paper with Fejér [14] we introduced the notion of monotonic convergence of a complex sequence $\left\{s_{n}\right\}$ to the limit $s$ requiring the monotonic convergence of the positive numbers $\left|s_{n}-s\right|$ to 0 . We showed that this property holds for the partial sums of any power series, provided that the coefficients are monotonic of the order 2. However, it does not hold generally for power series with only simply monotonic coefficients. More exactly we have proved

$$
|f(z)| \geqq\left|f(z)-s_{0}(z)\right| \geqq \cdots \geqq\left|f(z)-s_{n}(z)\right| \geqq \cdots \cdot
$$

As a consequence of this fact we obtain

$$
\left|f(z)-s_{n}(z)\right| \leqq|f(z)| \text { and hence }\left|s_{n}(x)\right| \leqq 2|f(z)| \text {. }
$$

A simple special case is that of the binomial series $f(z)=(1-z)^{-\rho}$ if $0<\rho<1$. The second inequality (36) corresponding to this case has been previously obtained, with a positive constant $A$ instead of 2, by Chapman [3] and M. Riesz [23]; it has importance for the summability theory of Fourier and Laplace series. As regards a direct proof see Szegö [32].

Assuming the so-called total monotony of the coefficients, the behaviour of the partial sums becomes still more regular. See Fejér [13].

Some of these results can be extended to integrals of the form 


$$
\int_{0}^{+\infty} f(t) e^{-x t} d t
$$

which have properties analogous to those of power series.

16. Conclusion. Numerous variations and analogs of the preceding problems are possible and some of them can be solved in a more or less exhaustive way. It would be a long task to give a complete account of this topic. Our intention was to set forth only the most typical results of this field, illustrating the way in which properties of an analytic function are reflected in the terms or in the sections of its power series development. A great many open problems in this direction can be formulated easily. Only a general analytic taste is able to decide which of these questions is worth being dealt with and to what extent they are connected with characteristic properties of analytic functions.

\section{BIBLIOGRAPHY}

1. C. Carathéodory, Über den Variabilitätsbereich der Fourierschen Konstanten von positiven harmonischen Funktionen, Rendiconti del Circolo matematico di Palermo, vol. 32 (1911), pp. 193-217.

2. C. Carathéodory and L. Fejér, Über den Zusammenhang der Extremen von harmonischen Funktionen mit ihren Koeffizienten und über den PicardLandauschen Satz, Rendiconti del Circolo matematico di Palermo, vol. 32 (1911), pp. 218-239.

3. S. Chapman, On non-integral orders of summability of series and integrals, Proceedings of the London Mathematical Society, (2), vol. 9 (1910), pp. 369409.

4. J. Dieudonné, Recherches sur quelques problèmes relatifs aux polynomes et aux fonctions bornées d'une variable complexe, Annales Scientifiques École Normale Supérieure, (3), vol. 48 (1931), pp. 247-358.

5. L. Fejér, Über gewisse Potenzreihen an der Konvergenzgrenze, Sitzungsberichte der math.-phys. Klasse der Bayerischen Akademie, 1910, Nr. 3, pp. $1-17$.

6. L. Fejér, Über gewisse durch die Fourier'sche und Laplace'sche Reihe definierte Mittelkurven und Mittelflächen, Rendiconti del Circolo matematico di Palermo, vol. 38 (1914), pp. 79-97.

7. L. Fejér, Über die Positivität von Summen, die nach trigonometrischen oder Legendre'schen Funktionen fortschreiten. (Erste Mitteilung.) Acta litterarum ac scientiarum Reg. Universitatis Hungaricae, sectio scient.-math., vol. 2 (1925), pp. 75-86.

8. L. Fejér, Über die Koeffizientensumme einer beschränkten und schlichten Potenzreihe, Acta Mathematica, vol. 49 (1926), pp. 183-190.

9. L. Fejér, Über die Grenzen der Abschnitte gewisser Potenzreihen, Acta litterarum ac scientiarum Reg. Universitatis Hungaricae, sectio scient.-math., vol. 4 (1928), pp. 14-24. 
10. L. Fejér, Einige Sätze, die sich auf das Vorzeichen einer ganzen rationalen Funktion beziehen; nebst Anwendungen dieser Sätze auf die Abschnitte und Abschnittsmittelwerte von ebenen und räumlichen harmonischen Entwicklungen und von beschränkten Potenzreihen, Monatshefte für Mathematik und Physik, vol. 35 (1928), pp. 305-344.

11. L. Fejér, Über ein trigonometrisches Analogon eines Kakeyaschen Satzes, Jahresbericht der Deutschen Mathematiker-Vereinigung, vol. 38 (1929), pp. 231-238.

12. L. Fejér, Neue Eigenschaften der Mittelwerte bei den Fourierreihen, Journal of the London Mathematical Society, vol. 8 (1933), pp. 53-62.

13. L. Fejér, Potenzreihen mit mehrfach monotoner Koeffizientenfolge und ihre Legendrepolynome, Proceedings of the Cambridge Philosophical Society, vol. 31 (1935), pp. 307-316.

14. L. Fejér and G. Szegö, Über die monotone Konvergenz von Potenzreihen mit mehrfach monotoner Koeffizientenfolge, Prace Matematyczno-Fizyczne, vol. 44 (1935), pp. 1-11.

15. L. Fejér, Trigonometrische Reihen und Potenzreihen mit mehrfach mono toner Koeffizientenfolge, Transactions of this Society, vol. 39 (1936), pp. 18-59.

16. E. Landau, Abschätzung der Koeffizientensumme einer Potenzreihe, Archiv der Mathematik und Physik, (3), vol. 21 (1913), pp. 42-50 and pp. 250255 ; vol. 24 (1916), pp. 250-260.

17. E. Landau, Über einen Fejérschen Satz, Nachrichten der Gesellschaft der Wissenschaften zu Göttingen, math.-phys. Klasse, 1925, p. 22.

18. V. Levin, Über die Koeffizientensummen einiger Klassen von Potenzreihen, Mathematische Zeitschrift, vol. 38 (1934), pp. 565-590.

19. J. E. Littlewood, On inequalities in the theory of functions, Proceedings of the London Mathematical Society, (2), vol. 23 (1924), pp. 481-519.

20. J. E. Littlewood and R. E. A. C. Paley, A proof that an odd schlicht function has bounded coefficients, Journal of the London Mathematical Society, vol. 7 (1932), pp. 167-169.

21. K. Noshiro, On the theory of schlicht functions, Journal of the Faculty of Science, Japanese Imperial University, (1), vol. 2 (1934), pp. 129-155.

22. G. Pick, Ein Abschätzungssatz für positive Nerwtonsche Potentiale, Jahresbericht der Deutschen Mathematiker-Vereinigung, vol. 23 (1915), pp. 329-332.

23. M. Riesz, Sur la sommation des séries de Fourier, Acta litterarum ac scientiarum Reg. Universitatis Hungaricae, sectio scient.-math., vol. 1 (1923), pp. 104-113.

24. W. Rogosinski, Über Bildschranken bei Potenzreihen und ihren Abschnitten, Mathematische Zeitschrift, vol. 17 (1923), pp. 260-276.

25. W. Rogosinski and G. Szegö, Über die Abschnitte von Potenzreihen, die in einem Kreise beschränkt bleiben, Mathematische Zeitschrift, vol. 28 (1928), pp. 73-94.

26. W. Rogosinski, Über positive harmonische Entwicklungen und typischreelle Potenzreihen, Mathematische Zeitschrift, vol. 35 (1932), pp. 93-121.

27. I. Schur, Über Potenzreihen, die im Innern des Einheitskreises beschränkt sind, Journal für Mathematik, vol. 147 (1917), pp. 205-232; vol. 148 (1918), pp. 122-145. 
28. I. Schur, Über die Koeffizientensummen einer Potenzreihe mit positivem reellen Teil, Archiv der Mathematik und Physik, (3), vol. 27 (1918), pp. 126135.

29. I. Schur and G. Szegö, Über die Abschnitte einer im Einheitskreise beschränkten Potenzreihe, Sitzungsberichte der preussischen Akademie, 1925, pp. 545-560.

30. S. Sidon, Ein Satz über positive harmonische Polynome, Jahresbericht der Deutschen Mathematiker-Vereinigung, vol. 35 (1926), pp. 97-99.

31. O. Szász, Ungleichungen für die Koeffizienten einer Potenzreihe, Mathematische Zeitschrift, vol. 1 (1918), pp. 163-183.

32. G. Szegö, Bemerkungen zu einer Arbeit von Herrn Fejér über die Legendreschen Polynome, Mathematische Zeitschrift, vol. 25 (1926), pp. 172-187.

33. G. Szegö, Koeffizientenabschätzungen bei ebenen und räumlichen harmonischen Entwicklungen, Mathematische Annalen, vol. 96 (1927), pp. 601-632.

34. G. Szegö, Korlatos hatvanysorokra vonatkozó újabb vizsgalatokról, Matematikai és Physikai Lapok, vol. 35 (1928), pp. 70-95.

35. G. Szegö, Zur Theorie der schlichten Abbildungen, Mathematische Annalen, vol. 100 (1928), pp. 188-211.

36. G. Szegö, Zur Theorie der Legendreschen Polynome, Jahresbericht der Deutschen Mathematiker-Vereinigung, vol. 40 (1931), pp. 163-166.

37. G. Szegö, Über räumliche harmonische Entwicklungen, welche in der Einheitskugel positiv sind, Monatshefte für Mathematik und Physik, vol. 43 (1936), pp. 148-156.

38. S. Takahashi, Über die Abschnitte einer ungeraden schlichten Potenzreihen, Proceedings of the Physico-Mathematical Society of Japan, (3), vol. 16 (1934), pp. 7-15.

39. O. Toeplitz, Über die Fouriersche Entwicklung positiver Funktionen, Rendiconti del Circolo matematico di Palermo, vol. 32 (1911), pp. 191-192.

WAShington UnIVERSity, St. Louis 